\title{
Implementing Subjective Global Assessment as a Routine Part of Dietitian Nutrition Assessments in a Multi-Site Health Care Organization Knowledge Translation at Providence Health Care
}

\section{Background}

Subjective global assessment (SGA) is the gold standard for diagnosing malnutrition. SGA is included in the Integrated Nutrition Pathway for Acute Care (INPAC) that is recommended by the Canadian Malnutrition Task Force (CMTF) for triaging nutrition care.

In 2017, a team of dietitians at Providence Health Care (PHC) embarked on a knowledge translation project to implement components of the INPAC pathway, including SGA. At that time, SGA was not consistently used by PHC dietitians to identify malnutrition.

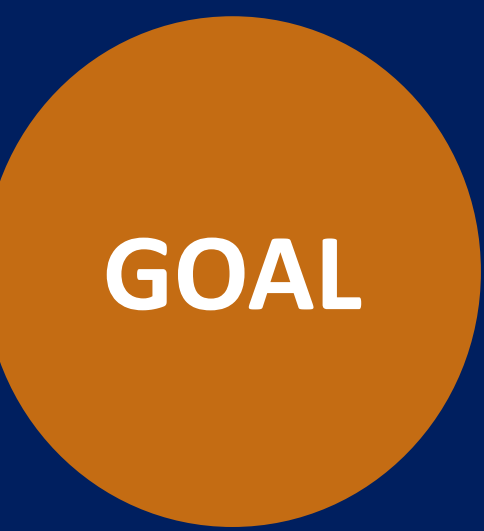

PHC Dietitians working in acute care use SGA to consistently identify and diagnose malnutrition

\section{Approach}

Implementation strategies included:

- Pre-training readings

- A group SGA workshop

- Hands-on training for peer champions with a CMTF SGA trainer

- Using peer champions to train dietetics students and additional staff

- Formatting the CMTF SGA form to meet PHC documentation requirements

- Embedding components of SGA into PHC Nutrition Care Plan forms

Evaluation strategies included:

- Surveys pre- and post-SGA workshop and hands-on training, and at 6 months postimplementation

- Chart audits

- Group experience sharing

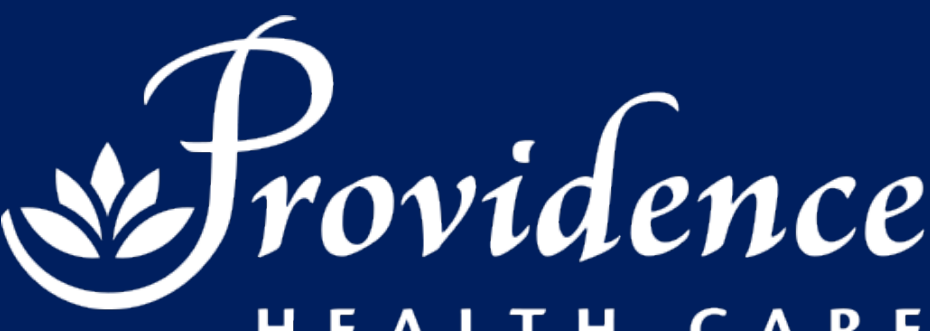

H E A L T H C A R

How you want to be treated.

\section{About Providence Health Care}

PHC has 593 acute care beds across three sites. PHC employs 65 dietitians of which 39 do some portion of their work in acute care.

\section{Outcomes}
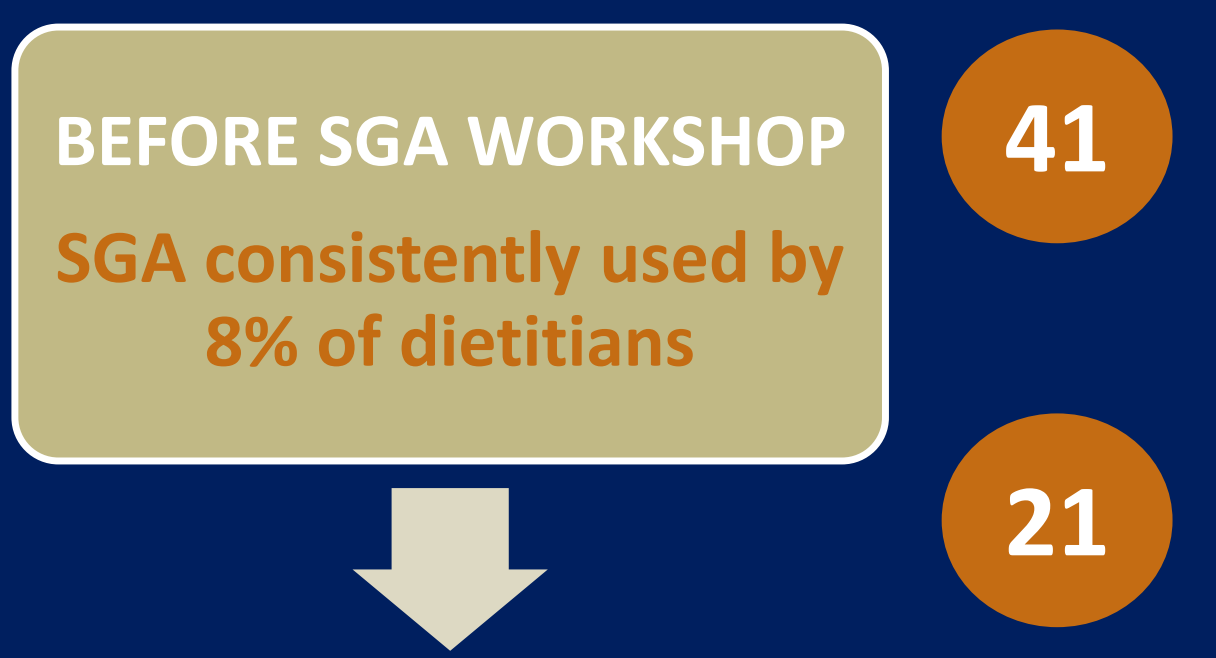

Dietitians participated in an SGA workshop

Dietitians received hands-on training with CMTF trainer
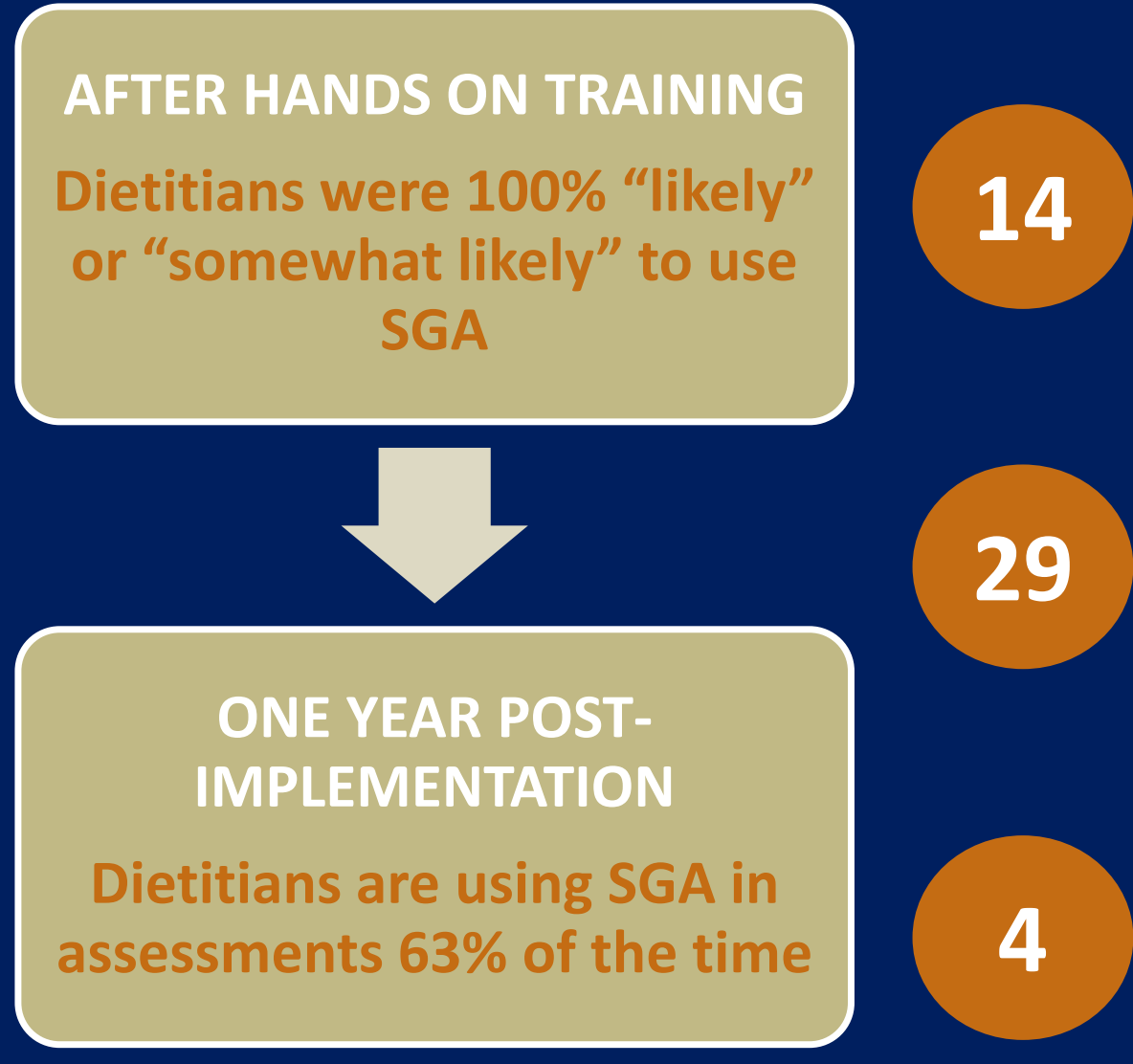

Dietitians were identified as peer champions

Dietitians received hands-on training with peer champion

Group experience

4. sharing sessions held

\section{Recommendations for Success}

- Identify all knowledge, skill and environmental barriers before, during and after implementation

- Use peer champions to for ongoing training needs

- Accommodate and encourage partnership between staff and peer champions to support ongoing training

- Provide re-training to solidify new knowledge and skill

- Embed practice change into organizational structures (e.g. forms, orientation, student placements)

- Continue group experience sharing as ongoing activity 